\title{
Abteilung Pharmakopöe der Swissmedic: Fachausschuss Komplementärmedizin neu gegründet
}

Die vermehrte Bedeutung der Komplementärmedizin in Europa wird zur Herausforderung der Arzneibücher, gilt es doch, die bewährten pharmazeutischen Regeln der Herstellpraxis so umzusetzen, dass sie für komplementärmedizinische Arzneimittel adäquat sind. Bisher versuchte der Fachausschuss Phytochemie nach Möglichkeit, diesen Auftrag zu erfüllen, doch es zeigte sich, dass die Experten die nötigen Kenntnisse nur beschränkt mitbrachten. Deshalb entschloss sich die Abteilung Pharmakopöe bei Swissmedic unter der Leitung von Tobias Gosdschan einen neuen Fachausschuss Komplementärmedizin zu gründen.

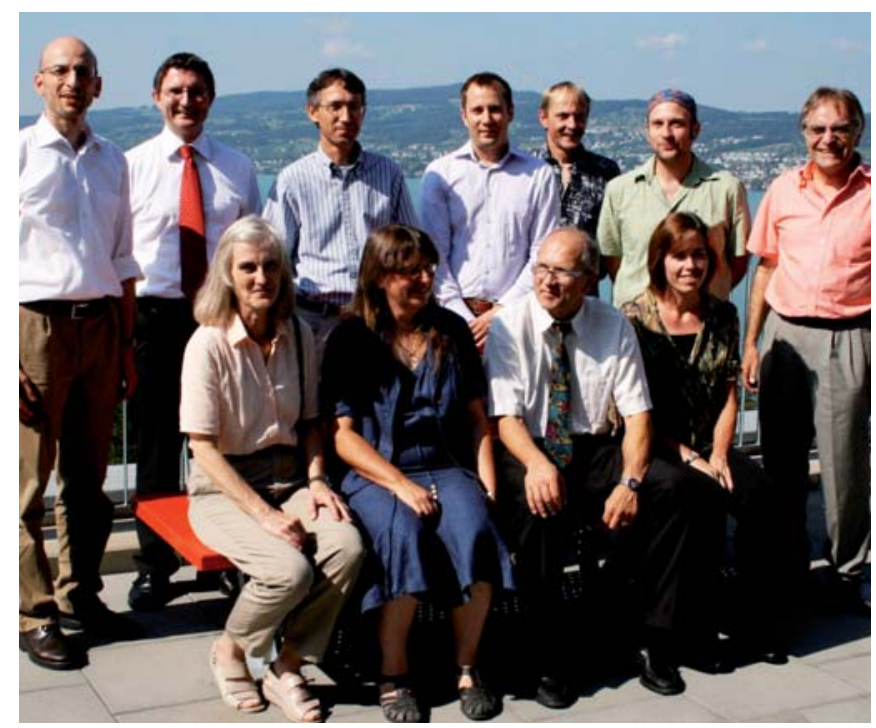

Der neue Fachausschuss vor den Gestaden des Zürichsees.

Stehend vinr: Jakob Meier, Weleda AG (Anthroposophische Medizin); Dr. Tobias Gosdschan, Swissmedic (Präsident Schweizerische Pharmakopoekommission); Dr. Richard Bolli, Swissmedic (Botanik, Regulatory Affairs); Dr. Romain Duc, Sérolab SA (Homöopathie); Thomas Lehmann, Swissmedic (Experte Analytik): Dr. Albert Blarer, Phytax AG (Traditionelle Chinesische Medizin); Prof.Dr. Beat Meier, Zürcher Hochschule angewandte Wissenschaften (Präsident des Fachausschusses).

Sitzend vinr: Dr. Silvia Trost, Swissmedic (Wissenschaftliche Mitarbeiterin); Margot Spohn, Swissmedic (Regulatory Affairs); Dr. Georg Zogg, Pharmavista GmbH (Homöopathie); Dr. Cornelia Huber (Swissmedic, Wissenschaftliche Betreuerin des Fachausschusses).

Auf dem Bild fehlen: Dr. Eike Reich, CAMAG (Expertin Dünnschichtchromaographie); Gabriela Gutknecht, Lian Chinaherb (Traditionelle Chinesische Medizin).

\section{Konstituierende Sitzung}

Am 27. August 2009 fand an der ZHAW (Zürcher Hochschule für angewandte Wissenschaften) in Wädenswil die konstituierende Sitzung des Ausschusses statt. Informiert wurde seitens Swissmedic über die Abläufe insbesondere im Zusammenhang mit der europäischen Pharmakopöe und über in die Vernehmlassung gehende, neu geschaffene Verfahren zur Aufnahme von Monographien jeglicher Art in die Schweizer Pharmakopöe. Am Beispiel von Sophorae Flos hatten die Experten auch schon einen Monographieentwurf der europäischen Pharmakopöe zu kommentieren und zu bearbeiten.

\section{Experten der Komplementärmedizin berufen}

In den Fachausschuss berufen wurden Experten der Komplementärmedizin. Sie vertreten einerseits die Richtungen Homöopathie, Traditionelle Chinesische Medizin und Anthroposophische Medizin, andererseits bringen sie Erfahrung aus der Zulassung, der Dünnschichtchromatographie und der Botanik von Arzneipflanzen mit. Die konstituierende Sitzung wurde mit einer Besichtigung der pharmazeutischen Labors an der ZHAW, einem Referat vom Präsidenten des Fachausschusses, Prof. Dr. Beat Meier, zu den in Wädenswil bisher geleisteten Arbeiten zum Thema "Zubereitungen in der Traditionellen Chinesischen Medizin” und einem Apéro abgeschlossen.

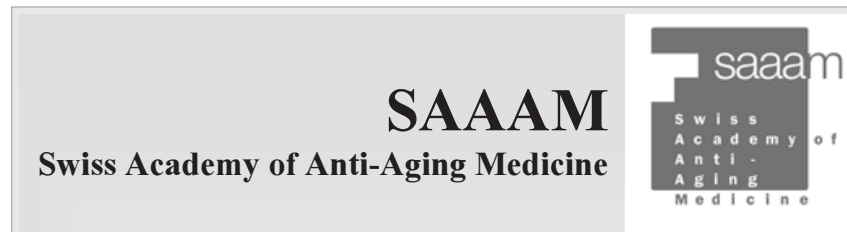

II. SICAAAM

II. Swiss International Congress on Anti-Aging and Aesthetic Medicine

GENEVA, 27.-29. November 2009

Ramada Park Hotel, Cointrin/Switzerland

The most scientific and academic conference in Europe on Integrative, Orthomolecular and Holistic Medicine

$$
\begin{gathered}
\text { 24 Credit Hours CME } \\
\text { 50+ Renowned Speakers } \\
\text { 17+Workshops }
\end{gathered}
$$

For more details and final programme, visit: www.saaam.org

\section{SAAAM}

Swiss Academy of Anti-Aging Medicine

11 Rue Rousseau • CH-1201 Geneva

Telephone +41 $229067773 \bullet \quad$ Fax +41229067778

www.saaam.org • info@saaam.org 